\title{
How combined aerobic training and pomegranate juice intake affect lipid profile? A clinical trial in men with type 2 diabetes
}

\author{
Sasan Nemati, Vahid Tadibi, Rastegar Hoseini \\ Department of Exercise Physiology, Faculty of Sport Sciences, Razi University, Kermanshah, Iran
}

\begin{abstract}
Study aim: This study aimed to investigate the effect of aerobic training (AT) and pomegranate juice intake (PJI) on the lipid profile in men with type 2 diabetes.

Materials and methods: This randomized clinical trial was performed in middle-aged men (40-50 years old) with type 2 diabetes. Participants were randomly assigned into four groups: AT + PJI $(n=9)$; AT $(n=10)$; PJI $(n=9)$, and control $(C)(n=10)$. The AT program consisted of $60-75 \%$ of $\mathrm{HR}_{\mathrm{Max}}, 40-60 \mathrm{~min} /$ day, three days/wk for eight weeks. Participants in the PJI group consumed $240 \mathrm{ml}$ of pomegranate juice (sugar or additive-free) daily for eight weeks. Lipid profile was measured at the beginning and end of the study. The data were analyzed through paired t-test and one-way analysis of variance, as well as Tukey's post hoc test at the signification level of $\mathrm{P}<0.05$.

Results: AT + PJI, PJI, and AT groups demonstrated significant improvements in lipid profile compared to the C group. The results show that the AT + PJI group had significantly lower TC and LDL ( $p=0.001$ and $p=0.002$, respectively), and significantly higher HDL $(\mathrm{p}=0.023)$ compared with the PJI group. There was no significant difference between AT and PJI groups. Also, TG was significantly lower in AT+ PJI compare to the C group.

Conclusions: AT + PJI is more effective than AT or PJI alone in the improvement of lipid profile in patients with type 2 diabetes.
\end{abstract}

Keywords: Exercise - Pomegranate - Diabetes Mellitus - Anthropometric Indices - Lipid Profile

\section{Introduction}

Diabetes, as a metabolic disorder, is mainly the elevation of blood glucose level that is associated with vascular complications leading to increased mortality [6]. Coronary artery disease, myocardial infarction, stroke, congestive heart failure, and peripheral vascular disease are among the cardiovascular complications in patients with diabetes $[6,18]$. As one of the main causes of death, the risk of developing these diseases in diabetes is two to four times higher among people with diabetes compared with healthy individuals and reduces life expectancy by five to ten years $[15,20]$. Hyperglycemia and lipid disorders are among the main etiology inducing structural heart damage; High levels of glucose lead to lipid disorders and accumulation of reactive oxygen species in myocardiocytes, which eventually leads to apoptosis or necrosis, and cell death [19]. About $11-44 \%$ of adults with diabetes have been shown to have lipid profile disorder with a $24-28 \%$ prevalence of hypercholesterolemia, and low serum HDL level that is also common in these patients [32]. It has been reported that improving the immune system via antioxidant therapy significantly reduces the severity of cardiovascular complications in diabetic patients [11]. In recent decades, exercise along with diet has been recommended as a good way to manage diabetes [22]. Aerobic training (AT) is one of the best non-pharmacological interventions in controlling blood glucose and lipid disorders [24]. Moderate-intensity AT has been suggested to improve the metabolic conditions of the diabetic person by strengthening the antioxidant system and reducing oxidative factors [28].

Oxidative stress is the result of an imbalance between the production of free radicals and the antioxidants that increased in diabetes [19]. One of the factors that lead to increased oxidative stress is hyperglycemia [19, 29]. Clinical evidence also supports the antioxidant role of pomegranate in the prevention and treatment of diabetes $[4,13]$. The antidiabetic effects of pomegranate are due to the presence of oleanolic, ursolic, and gallic acids [17]. Pomegranate extract seems to have more polyphenols than many beverages [23]. The most distinctive feature of all flavonoids is acting 
as an antioxidant $[21,23]$. The antioxidant effects of pomegranate juice $(\mathrm{PJ})$ reduce the ischemic heart disease risk factors by inhibiting the oxidation of low-density lipoprotein, reducing the formation of foam cells, and reducing the oxidation of macrophages [25, 31].

Therefore, using affordable and accessible methods to control and treat diabetes seems to be plausible, with regards to the growing prevalence, subsequent heart complications, and high treatment costs. Some studies have investigated the separate effects of AT and pomegranate juice intake (PJI) on patients with type 2 diabetes. However, to our knowledge, the simultaneous effect of AT and PJI has not been investigated in patients with type 2 diabetes. Considering the effect of PJI as well as AT on improving insulin resistance and lipid disorders, this study was performed to investigate separate and the simultaneous effect of AT and PJI on lipid profile in men with diabetes.

\section{Materials and methods}

\section{Participants}

In this randomized clinical trial study, 40 men with type 2 diabetes were recruited by a voluntary sampling method and by referring to specialized diabetes clinics. The sample size for each group was estimated to be 10 , using $\mathrm{G}^{*}$ Power software with $\alpha=0.05$ and statistical power of $80 \%$. Participants were randomly assigned into four groups including AT + PJI $(n=10)$, AT $(n=10)$, PJI $(n=10)$, and control (C) group $(n=10)$. It is worth mentioning, one participant in the AT + PJI group and one subject in the PJI group refused to participate in the intervention program (figure 1). The present study was approved by the Committee of Ethics in Research at Kermanshah University of Medical Sciences and registered

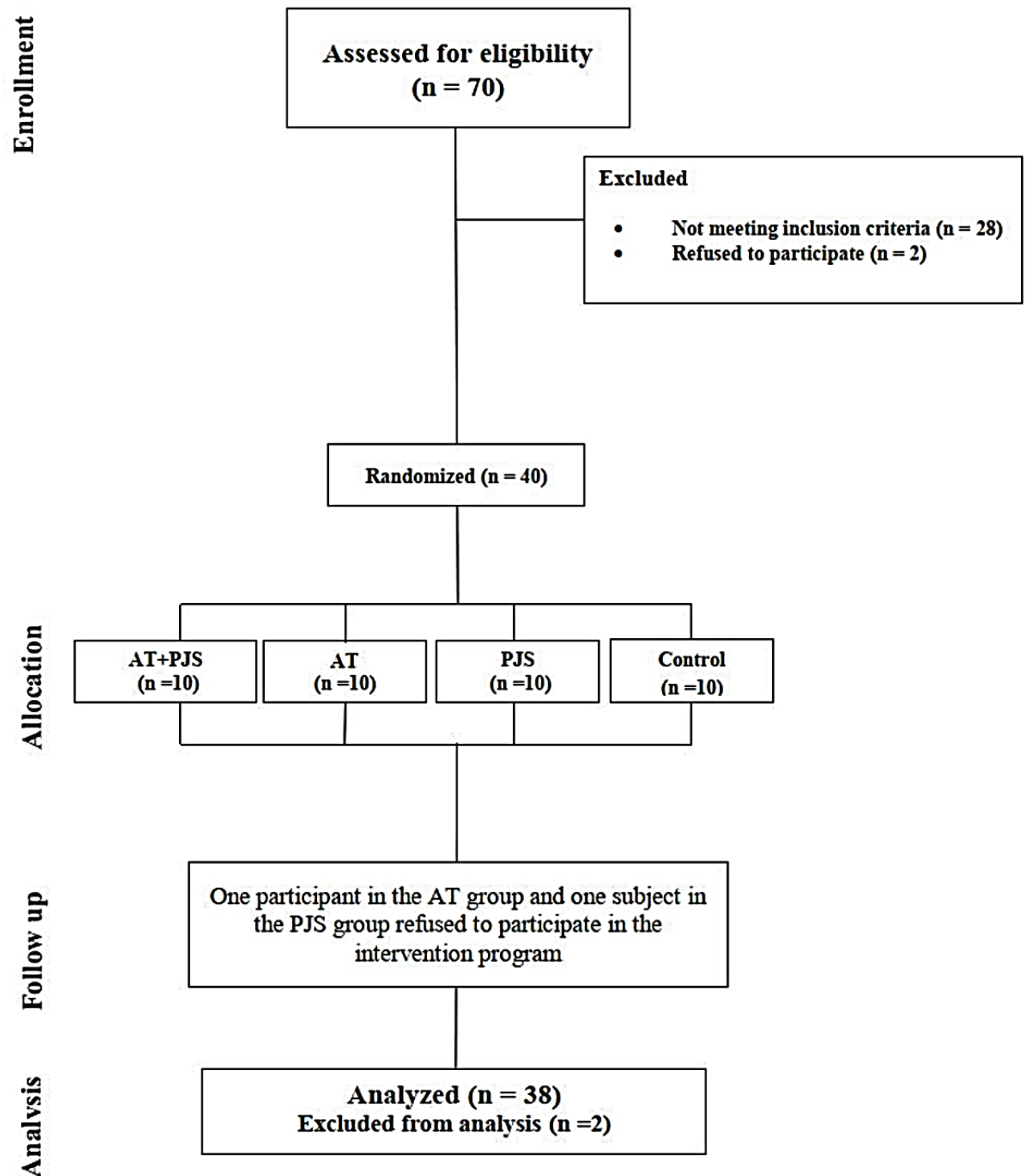

Figure 1. Diagram of the progress through the phases of the parallel randomized trial of four groups (enrolment, intervention allocation, follow-up, and data analysis) 
Table 1. Aerobic training protocol

\begin{tabular}{|c|c|c|c|c|c|c|c|c|}
\hline & \multicolumn{8}{|c|}{ Week } \\
\hline Variables & 1 & 2 & 3 & 4 & 5 & 6 & 7 & 8 \\
\hline Intensity $\left[\mathrm{HR}_{\mathrm{Max}}\right]$ & $60-65 \%$ & $60-65 \%$ & $65-70 \%$ & $65-70 \%$ & $65-70 \%$ & $70-75 \%$ & $70-75 \%$ & $70-75 \%$ \\
\hline Time [min] & 40 & 40 & 50 & 50 & 50 & 60 & 60 & 60 \\
\hline
\end{tabular}

in the Iranian Clinical Trial Registration Center under the code IRCT20200907048650N1. Before the study, informed written consent was obtained from all participants after a comprehensive oral and written explanation of the study was provided to them. All patients were allowed to leave the study at any point, voluntarily.

\section{Study design}

Questionnaires of cooperation and personal information, physical activity readiness questionnaire (PAR-Q), and informed consent were provided from all participants. Inclusion criteria included: men with type 2 diabetes and no other chronic disease or musculoskeletal problems, aged 40-50 years, fasting blood sugar above $126 \mathrm{mg}$, taking common diabetes medications such as metformin and glibenclamide, no insulin injection, body mass index above 24.99, no smoking, and lack of regular exercise for at least last six months before the beginning of the study. Participants were fully acquainted with the aims of the study and received the necessary oral and written explanation.

\section{Procedures}

After the informed consent was signed, the volunteers were examined by a specialist in endocrinology and metabolism. On the first day, height was measured to the nearest $0.5 \mathrm{~cm}$ using a stadiometer (DETECTO, Model 3PHTROD-WM, USA). Bodyweight (BW), Body mass index (BMI), waist-hip ratio (WHR), and body fat percentage (BFP) were obtained by a bioelectric impedance body composition analyzer (Zeus 9.9 PLUS; Jawon Medical Co., Ltd., Kungsang Bukdo, South Korea) in the fasting state.

\section{Aerobic training}

The 8-week AT program was consisted of a total of 40-60 min jogging and running at $60-75 \%$ of maximum heart rate (HRmax) (Table1). The AT program was based on previous studies and recommendations from the American Diabetes Association (ADA) $[33,34]$. AT consists of three phases: warm-up (10 $\mathrm{min})$, AT (20-40 $\mathrm{min})$, and cool down (10 min). At baseline, the training phase commenced with 40 minutes of jogging at $65 \%$ of HRmax in the first week and increased to 60 -minute of running at $75 \%$ of HRmax by the final week. To assure that the desired heart rate (exercise intensity) was achieved and maintained during the AT phase, each participant underwent heart-rate monitoring with a polar heart rate monitor (model: FT1) and using the 6-20 rating of perceived exertion (RPE) scale (Table 1) [12]. The HRmax formula was used to determine the target heart rate $\left[\mathrm{HR}_{\max }=220-\right.$ age $]$.

\section{Pomegranate juice intake}

Participants in both PJI consumed $240 \mathrm{ml}$ of pure PJ from Orum Narin Company with SHADLEE brand (without sugar and other additives) daily after lunch for eight weeks.

To prepare the PJ, the pomegranates were picked by hand and after washing, the pomegranate seeds were separated from the skin and the meat inside it by a specific machine and stored in the tank. Then the pomegranates were pressed, juiced, and pasteurized at $80^{\circ} \mathrm{C}$. Concentrated PJ was produced after enzymatic, fining, and filtration steps. Finally, it was packed and transported to a cool warehouse. The PJ contained $20 \%$ PJ concentrate and no additives were added.

Participants in the AT and C groups received a waterbased placebo with the same shape, color, and smell as the PJI, during the study [30]. Before attending the training program, the participants filled two questionnaires. First, a questionnaire was determined to assess the readiness of participants, including demographic data, health status, current alcohol intake, and physical activity. A second questionnaire was a detailed semi-quantitative food frequency questionnaire adapted to the Iranian population and composed everyday food items, serving sizes, and meals, as designed to record and analyze 3-day food, recalls, before, and at the end of the intervention. To determine the food intake and the amount of macronutrient consumption (protein, fat, and carbohydrates), the Food Processor nutritionist four software (FPN4) was used. Subjects were asked to consume the same food and macronutrient composition one day before collecting blood samples in the pre and post-test. In general, the subject's diet consisted of $55 \%$ carbohydrates, $30 \%$ fat, and $15 \%$ protein. 
Table 2. Mean \pm SD demographic information and anthropometric indices before the intervention among the groups

\begin{tabular}{lccccc}
\hline Variables & AT + PJI $(\mathrm{n}=9)$ & AT $(\mathrm{n}=10)$ & PJI $(\mathrm{n}=9)$ & $\mathrm{C}(\mathrm{n}=10)$ & P-value $_{\mathrm{a}}$ \\
\hline Age [years] & $43.22 \pm 2.48$ & $42.60 \pm 1.89$ & $41.33 \pm 1.50$ & $41.81 \pm 1.88$ & 0.336 \\
Height $[\mathrm{cm}]$ & $162 \pm 54.18$ & $166 \pm 5.45$ & $164.55 \pm 4.79$ & $164.45 \pm 5.78$ & 0.393 \\
Body weight $[\mathrm{kg}]$ & $90.44 \pm 4.92$ & $88.80 \pm 3.35$ & $88.44 \pm 2.50$ & $90.09 \pm 4.20$ & 0.537 \\
BMI $\left[\mathrm{kg} / \mathrm{m}^{2}\right]$ & $34.55 \pm 3.14$ & $32.28 \pm 1.78$ & $32.69 \pm 1.30$ & $33.34 \pm 1.51$ & 0.112 \\
FBS $[\mathrm{mg} / \mathrm{dl}]$ & $144.44 \pm 3.12$ & $145.10 \pm 3.84$ & $146.88 \pm 7.42$ & $144.90 \pm 7.24$ & 0.802 \\
Insulin $[\mu \mathrm{U} / \mathrm{ml}]$ & $7.30 \pm 0.70$ & $7.31 \pm 0.86$ & $7.06 \pm 0.75$ & $7.30 \pm 0.78$ & 0.832 \\
\hline
\end{tabular}

$\mathrm{AT}+\mathrm{PJI}-$ Aerobic training + pomegranate juice intake group; AT - Aerobic training group; PJI - pomegranate juice intake group; C - control group; P values with superscript " $\mathrm{a}$ " are calculated using a one-way analysis of variance test.

\section{Blood sampling}

Blood samples were taken twice, a day before the first training session (pre-test) and all subjects arrived at 8:00 a.m. on the day of the test in the clinical laboratory after more than 12 hours fasting. After maintaining the subjects in a stable state for 30 minutes, $20 \mathrm{ml}$ of venous blood was sampled from the antecubital vein using an anticoagulant-treated syringe. The sampled blood was placed in a tube that was not treated for anticoagulation and was then centrifuged at 3,000 rpm using a centrifugal separator for 10 minutes. After extracting the serum from the cellular components, the serum was put in a storage tube and stored in the refrigerator at $-70 \square$ until analysis. Blood lipid profile (Triglycerides (TG), total cholesterol (TC), high-density lipoprotein (HDL), and low-density lipoprotein (LDL)) were measured enzymatically with Hitachi Kit, Tokyo, Japan, and Glucose with the enzymatic method (Pars Azmoon kit made in Iran). Fasting blood sugar was calculated by enzymatic method using Pars Azmoon test kits (Iran), insulin by ELISA method using Mercoda Company kit (Sweden), and Insulin resistance index based on the following formula [17].

\section{Statistical analysis}

The results obtained in this study were analyzed using IBM SPSS (version 24.0) at the significance level of $\alpha=0.05$. The descriptive statistics quantity was presented as the mean and the standard error of the mean (SE). The Shapiro-Wilk's test was used for evaluating the normality of distribution. To compare the mean hepatic risk factors between and within groups, one-way ANOVA and t-test were used, respectively; Tukey's post hoc test was used if significant differences were found.

\section{Results}

The findings on some demographic information, fasting blood sugar (FBS), and insulin of the participants and their between-group comparison are presented in Table 2 .

The results of the one-way ANOVA test showed no significant difference in demographic information, FBS, and insulin between the groups.

TC, TG, LDL, and HDL levels improved significantly from pre-test to post-test within all experimental groups, as detailed in Figures 2, 3, 4, and 5. The results of oneway ANOVA showed no significant difference in TC, TG, LDL, and HDL between the groups in the pre-test, however in the post-test, there was a significant difference in the above variables between the groups. Lipid Profile changes were significant in AT + PJI, AT, and PJI compared to $\mathrm{C}$.

Figures 2, 3, 4, and 5 show that the AT + PJI had significantly lower TC, LDL $(p=0.001$, and $p=0.002$, respectively), and significantly higher HDL $(p=0.023)$ compared with PJI. Also, the results of this study showed no significant difference between AT and PJI groups. Also, TG was significantly lower in AT + PJI compare to C, but not in AT and PIJ alone.

\section{Discussion}

Findings of the present study showed that after 8 weeks of the interventional program AT+PJI the lowest levels of TC, TG, LDL, and the highest levels of HDL were observed in the AT+PJI; Therefore, AT + PJI had been more effective in improving blood lipid profile compared to 


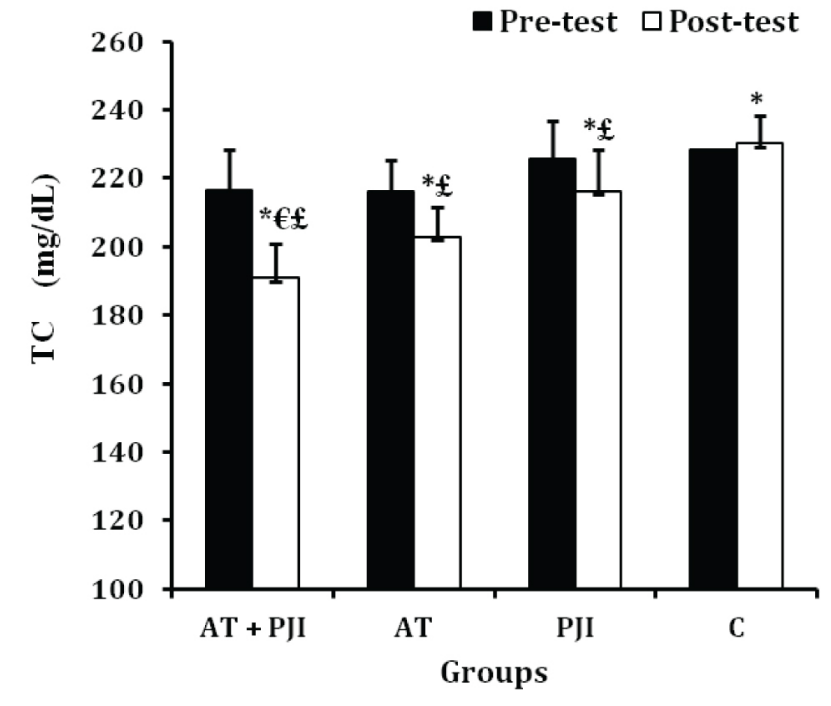

Figure 2. Comparison between mean $\pm \mathrm{SD}$ of $\mathrm{TC}$ between groups

$\mathrm{AT}+\mathrm{PJI}-$ Aerobic training + pomegranate juice intake group; AT Aerobic training group; $\mathrm{PJI}$ - pomegranate juice intake group; $\mathrm{C}$ - control group; TC - Total Cholesterol; *: Significantly different compared to pre-test within the groups; $£$ : Significantly different compared to C; $€$ : Significantly different compared to PJI.

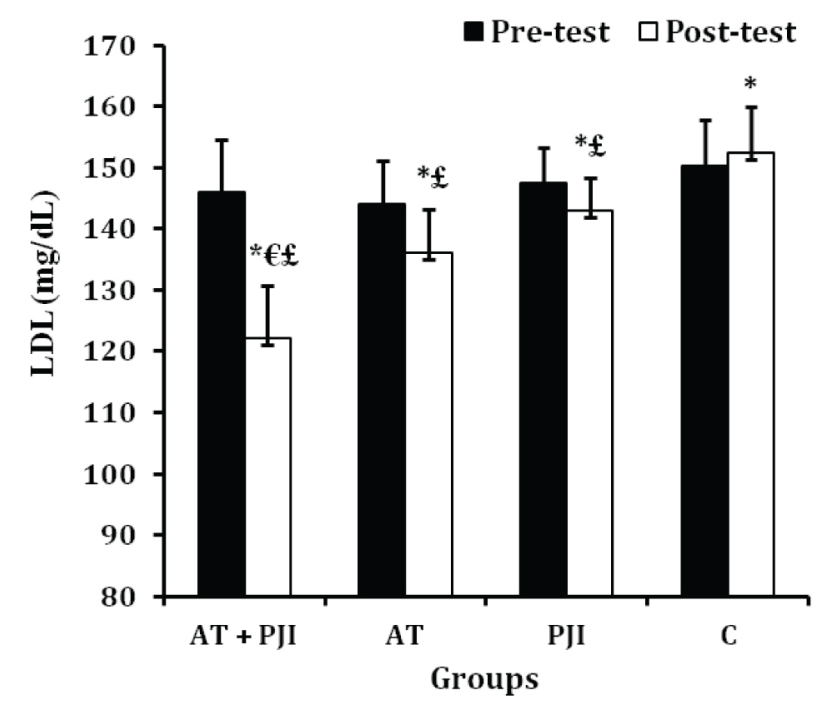

Figure 4. Comparison between mean $\pm \mathrm{SD}$ of LDL between groups

$\mathrm{AT}+\mathrm{PJI}-$ Aerobic training + pomegranate juice intake group; AT $-\mathrm{Ae}-$ robic training group; PJI - pomegranate juice intake group; $\mathrm{C}$ - control group; LDL - Low-Density Lipoprotein; *: Significantly different compared to pre-test within the groups; $£$ : Significantly different compared to $C ; €$ : Significantly different compared to PJI.

PJI or AT alone. In Contrast, Kaplan et al. [14] found that two months of PJI did not affect TC levels significantly in rats [14]. Aviram et al. [1] investigated the anti-atherogenic effect of various pomegranate-based supplements in atherosclerotic mice with impaired apolipoprotein E production and concluded that $\mathrm{PJ}$ and pomegranate

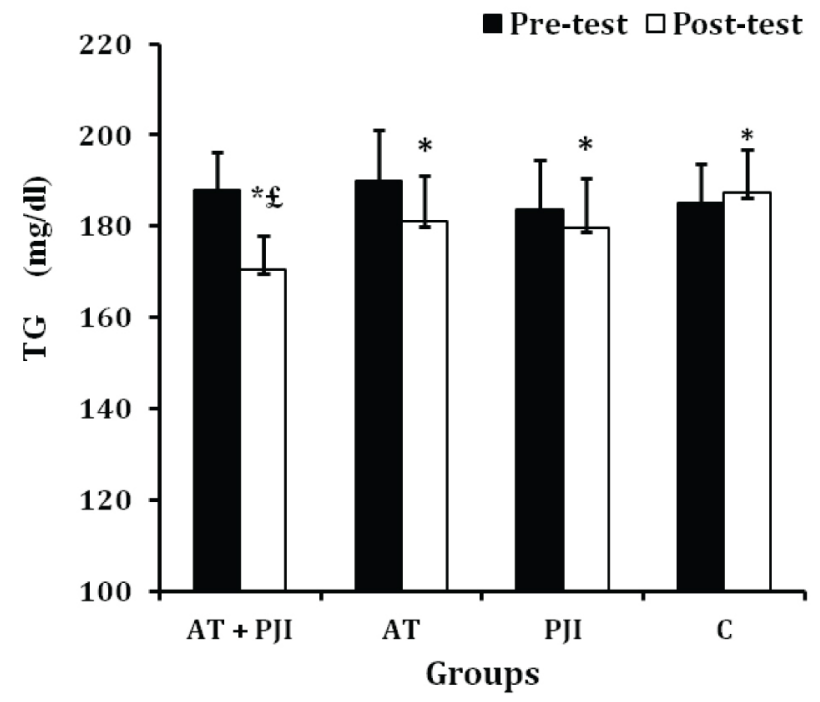

Figure 3. Comparison between mean $\pm \mathrm{SD}$ of TG between groups

AT + PJI - Aerobic training + pomegranate juice intake group; AT - Aerobic training group; PJI - pomegranate juice intake group; $\mathrm{C}$ - control group; TG - Triglycerides; *: Significantly different compared to pre-test within the groups; $£$ : Significantly different compared to $\mathrm{C}$.

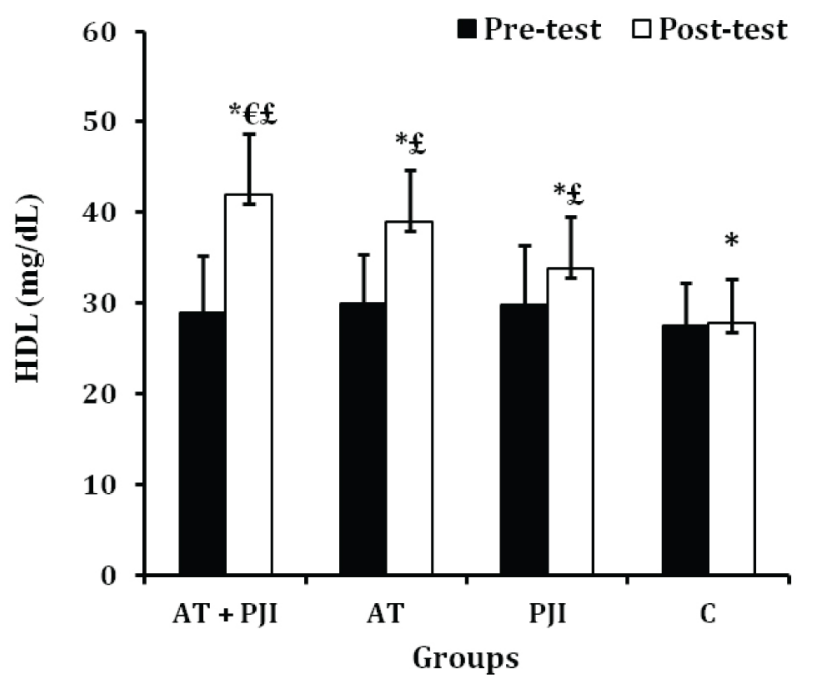

Figure 5. Comparison between mean \pm SD of HDL between groups

$\mathrm{AT}+\mathrm{PJI}-$ Aerobic training + pomegranate juice intake group; AT - Aerobic training group; $\mathrm{PJI}$ - pomegranate juice intake group; $\mathrm{C}$ - control group; HDL - High-Density Lipoprotein; *: Significantly different compared to pre-test within the groups; £: Significantly different compared to C; $€$ : Significantly different compared to PJI.

flower extract had the most reducing effect on atherosclerotic plaque formation by $34 \%$ and $70 \%$, respectively [1]. The results of Rajabian et al. [27] study showed that the PJ or seed oil was not effective in reducing TC, TG, LDL, and VLDL; however, it significantly prevented the development of aorta atherosclerotic plaques formation 
in treated hypercholesterolemic rabbits [27]. Rosenblatt et al (2006) found no significant effect on TC and TG levels in diabetic patients after consuming $50 \mathrm{ml} /$ day of PJ for 3 months [29]. Esmailzadeh et al. [7] Showed that consumption of $40 \mathrm{~g}$ of concentrated PJ per day for 8 weeks in diabetic patients, significantly reduced TC and LDL, while was not significantly affecting serum TG and HDL [7]. Several studies have shown that PJ contains flavonoids compounds that could improve the lipid profile due to its antioxidant feature [7; 37]. Human and cellular studies have shown that PJ can increase the expression and activity of paraoxonase- 1 . This enzyme has antioxidant properties that prevent the oxidation of LDL-C and HDL-C [2, 35]. PJ increases paraoxonase- 1 activity by reducing fat peroxides or directly affecting the enzyme [8]. Increased serum activity of this enzyme in diabetics, decreases hypercholesterolemia, and cardiovascular complications $[2,8]$. On the other hand, reducing cholesterol absorption, increasing cholesterol excretion, and modulating $\mathrm{HMG}-\mathrm{COA}$ reductase and acyltransferase (two key enzymes in cholesterol metabolism) are the other impacts of PJ [7, 35]. The PJ also inhibits HDL depletion by stimulating the release of HDL from macrophage cells [14]. Numerous studies have shown the beneficial effects of AT in improving lipid profile $[9,16,38]$. For example, Kasterswannack et al. (2015) observed that AT decreased TC, TG, and LDL, and increased HDL [16]. Increased fat oxidation as an energy substrate, both during exercise and recovery, are the main mechanisms of AT in improving the lipid profile. Other studies also showed that AT increases the amount of type A lipoprotein and the enzyme lipoprotein lipase (LPL) and ultimately decreases LDL $[3,16]$. Therefore, the possible mechanism by which AT + PJI reduces TC and TG levels might be explained by the increased lipoprotein lipase response and activity. Gordon et al. (2016) observed that subjects with regular physical activity have significantly higher HDL concentrations than non-physically active subjects [10]. According to the results of studies, AT increases HDL by increasing the activity of lipoprotein lipase enzyme and hydrolyzing plasma triglycerides, which have protective effects against heart disease $[10,26]$.

\section{Conclusions}

Based on the findings of the present study, eight weeks of AT or PJI has positive effects on the lipid profile in patients with type 2 diabetes. However, consumption of PJ in conjunction with AT would have more positive effects on lipid profile in these patients.
Conflict of interest: Authors state no conflict of interest.

\section{References}

1. Aviram M. Dornfeld L. (2001) Pomegranate juice consumption inhibits serum angiotensin converting enzyme activity and reduces systolic blood pressure. Atherosclerosis, 158(1):195-198.

2. Aviram M., Rosenblat M., Gaitini D., Nitecki S., Hoffman A., Dornfeld L., Volkova N., Presser D., Attias J. Liker H. (2004) Pomegranate juice consumption for 3 years by patients with carotid artery stenosis reduces common carotid intima-media thickness, blood pressure and LDL oxidation. Clin. Nutr., 23(3): 423-433.

3. Babaei P., Damirchi A. Hoseini R. (2015) The interaction effects of aerobic exercise training and vitamin D supplementation on plasma lipid profiles and insulin resistance in ovariectomized rats. J. Exerc. Nutrition Biochem., 19(3): 173-178.

4. Basuny A.M. Mohammed G.M. (2020) Anti-diabetic and Antioxidant Effects of Olive and Pomegranate Leave Juice on Streptozotocin Diabetes in Rats. J. Res. Lepid., 51(3): 141-156.

5. Cassidy S., Thoma C., Houghton D. Trenell M.I. (2017) High-intensity interval training: a review of its impact on glucose control and cardiometabolic health. Diabetologia, 60(1): 7-23.

6. Dal Canto E., Ceriello A., Rydén L., Ferrini M., Hansen T.B., Schnell O., Standl E. Beulens J.W. (2019) Diabetes as a cardiovascular risk factor: An overview of global trends of macro and micro vascular complications. Eur. $J$. Prev. Cardiol., 26(2_suppl): 25-32.

7. Esmaillzadeh A., Tahbaz F., Gaieni I., Alavi-Majd H. Azadbakht L. (2006) Cholesterol-lowering effect of concentrated pomegranate juice consumption in type II diabetic patients with hyperlipidemia. Int. J. Vitam. Nutr. Res., 76(3): 147-151.

8. Estrada-Luna D., Martinez-Hinojosa E., Cancino-Diaz J., Belefant-Miller H., Lopez-Rodriguez G. Betanzos-Cabrera G. (2018) Daily supplementation with fresh pomegranate juice increases paraoxonase 1 expression and activity in mice fed a high-fat diet. Eur. J. Nutr., 57(1): 383-389.

9. Fikenzer K., Fikenzer S., Laufs U. Werner C. (2018) Effects of endurance training on serum lipids. Vasc. Pharmacol., 101(1): 9-20.

10. Gordon B., Chen S. Durstine J.L. (2014) The effects of exercise training on the traditional lipid profile and beyond. Curr. Sports Med. Rep., 13(4): 253-259.

11. Gu J., Yan X., Dai X., Wang Y., Lin Q., Xiao J., Zhou S., Zhang J., Wang K. Zeng J. (2018) Metallothionein pre- 
serves Akt2 activity and cardiac function via inhibiting TRB3 in diabetic hearts. Diabetes, 67(3): 507-517.

12. Heil D.P. (2001) ACSM's guidelines for exercise testing and prescription. Medicine and Science in Sports and Exercise., 33(2): 343-348.

13. Hou C., Zhang W., Li J., Du L., Lv O., Zhao S., Li J. (2019) Beneficial effects of pomegranate on lipid metabolism in metabolic disorders. Mol. Nutr. Food Res., 63(16): 1-12.

14. Kaplan M., Hayek T., Raz A., Coleman R., Dornfeld L., Vaya J. Aviram M. (2001) Pomegranate juice supplementation to atherosclerotic mice reduces macrophage lipid peroxidation, cellular cholesterol accumulation and development of atherosclerosis. J. Nutr., 131(8): 2082-2089.

15. Kerimi A., Nyambe-Silavwe H., Gauer J.S., TomásBarberán F.A., Williamson G. (2017) Pomegranate juice, but not an extract, confers a lower glycemic response on a high-glycemic index food: Randomized, crossover, controlled trials in healthy subjects. Am. J. Clin. Nutr., 106(6): 1384-1393.

16. Kostrzewa-Nowak D., Nowak R., Jastrzębski Z., Zarębska A., Bichowska M., Drobnik-Kozakiewicz I., Radzimiński Ł., Leońska-Duniec A., Ficek K. Cięszczyk P. (2015) Effect of 12-week-long aerobic training programme on body composition, aerobic capacity, complete blood count and blood lipid profile among young women. Biochem. Med., 25(1): 103-113.

17. Kumar A.S., Maiya A.G., Shastry B., Vaishali K., Ravishankar N., Hazari A., Gundmi S. Jadhav R. (2019) Exercise and insulin resistance in type 2 diabetes mellitus: A systematic review and meta-analysis. Ann. Phys. Rehabil. Med., 62(2): 98-103.

18. Liang W. dong Ye D. (2019) The potential of adipokines as biomarkers and therapeutic agents for vascular complications in type 2 diabetes mellitus. Cytokine Growth Factor Rev., 48: 32-39.

19. Liu Q., Wang S. Cai L. (2014) Diabetic cardiomyopathy and its mechanisms: role of oxidative stress and damage. J. Diabetes Investig., 5(6): 623-634.

20. Long A.N. Dagogo-Jack S. (2011) Comorbidities of diabetes and hypertension: mechanisms and approach to target organ protection. J. Clin. Hypertens., 13(4): 244-251.

21. Manthou E., Georgakouli K., Deli C.K., Sotiropoulos A., Fatouros I.G., Kouretas D., Haroutounian S., Matthaiou C., Koutedakis Y. Jamurtas A.Z. (2017) Effect of pomegranate juice consumption on biochemical parameters and complete blood count. Exp. Ther. Med., 14(2): 1756-1762.

22. Matos M., Mendes R., Silva A.B. Sousa N. (2018) Physical activity and exercise on diabetic foot related outcomes: a systematic review. Diabetes Res. Clin. Pract., 139: $81-90$
23. Matthaiou C.M., Goutzourelas N., Stagos D., Sarafoglou E., Jamurtas A., Koulocheri S.D., Haroutounian S.A., Tsatsakis A.M. Kouretas D. (2014) Pomegranate juice consumption increases GSH levels and reduces lipid and protein oxidation in human blood. Food Chem. Toxicol., 73(2): 1-6.

24. McDonald M.W., Olver T.D., Dotzert M.S., Jurrissen T.J., Noble E.G., Padilla J. Melling C.J. (2019) Aerobic exercise training improves insulin-induced vasorelaxation in a vessel-specific manner in rats with insulin-treated experimental diabetes. Diabetes Vasc. Dis. Res., 16(1): 77-86.

25. Minaiyan M., Zolfaghari B., Taheri D. Gomarian M. (2014) Preventive effect of three pomegranate (Punica granatum L.) seeds fractions on cerulein-induced acute pancreatitis in mice. Int. J. Prev. Med., 5(4): 394.

26. Pawar A.A. Mane D.A. (2018) Study of association between calcium and lipid profile with respect to menopause. Indian J. Public Health Res. Dev., 9(10): 158-162.

27. Radjabian T., Fallah Husseini H., Karami M., Rasooli I. Faghihzadeh S. (2008) Effect of Pomegranate Fruit Juice and Seed Oil on Serum Lipid Levels and Atherosclerosis Development in Hypercholesterolemic Rabbits. J. Med. Plants, 1(25): 93-104.

28. Riddell M.C., Zaharieva D.P., Tansey M., Tsalikian E., Admon G., Li Z., Kollman C. Beck R.W. (2019) Individual glucose responses to prolonged moderate intensity aerobic exercise in adolescents with type 1 diabetes: The higher they start, the harder they fall. Pediatr. Diabetes, 20(1): 99-106.

29. Rosenblat M., Hayek T. Aviram M. (2006) Anti-oxidative effects of pomegranate juice (PJ) consumption by diabetic patients on serum and on macrophages. Atherosclerosis, 187(2): 363-371.

30. Sahebkar A., Gurban C., Serban A., Andrica F. Serban M.-C. (2016) Effects of supplementation with pomegranate juice on plasma C-reactive protein concentrations: A systematic review and meta-analysis of randomized controlled trials. Phytomedicine, 23(11): 1095-1102.

31. Salwe K.J., Sachdev D.O., Bahurupi Y. Kumarappan M. (2015) Evaluation of antidiabetic, hypolipedimic and antioxidant activity of hydroalcoholic extract of leaves and fruit peel of Punica granatum in male Wistar albino rats. J. Nat. Sci. Biol. Med., 6(1): 56-65.

32. Santos H.O., Bueno A.A. Mota J.F. (2018) The effect of artichoke on lipid profile: A review of possible mechanisms of action. Pharmacol. Res., 137: 170-178.

33. Sanz C., Gautier J.-F. Hanaire H. (2010) Physical exercise for the prevention and treatment of type 2 diabetes. Diabetes Metab., 36(5): 346-351.

34. Sigal R.J., Kenny G.P., Wasserman D.H. CastanedaSceppa C. (2004) Physical activity/exercise and type 2 diabetes. Diabetes Care, 27(10): 2518-2539. 
35. Sohrab G., Roshan H., Ebrahimof S., Nikpayam O., Sotoudeh G. Siasi F. (2019) Effects of pomegranate juice consumption on blood pressure and lipid profile in patients with type 2 diabetes: A single-blind randomized clinical trial. Clin. Nutr. ESPEN., 29 (1): 30-35.

36. Støa E.M., Meling S., Nyhus L.-K., Strømstad G., Mangerud K.M., Helgerud J., Bratland-Sanda S. Støren Ø. (2017) High-intensity aerobic interval training improves aerobic fitness and $\mathrm{HbA1c}$ among persons diagnosed with type 2 diabetes. Eur. J. Appl. Physiol., 117(3): 455-467.

37. Stowe C.B. (2011) The effects of pomegranate juice consumption on blood pressure and cardiovascular health. Complement. Ther. Clin. Pract., 17(2): 113-115.

38. Theodorou A.A., Panayiotou G., Volaklis K.A., Douda H.T., Paschalis V., Nikolaidis M.G., Smilios I.,
Toubekis A., Kyprianou D. Papadopoulos I. (2016) Aerobic, resistance and combined training and detraining on body composition, muscle strength, lipid profile and inflammation in coronary artery disease patients. Res. Sports Med., 24(3): 171-184.

\section{Received 03.12.2020 \\ Accepted 23.03.2021}

(C) University of Physical Education, Warsaw, Poland

\section{Acknowledgments}

The authors would like to thank the subjects for their willing participation in this study. 